\title{
INFLUENCE OF TEMPERATURE OF ION IMPLAN- TATION ON DISTRIBUTION OF CONCENTRATION OF DOPANT IN AN IMPLANTED-JUNCTION RECTIFIERS
}

\author{
E.L. Pankratov ${ }^{1}$, E.A. Bulaeva ${ }^{1,2}$ \\ ${ }^{1}$ Nizhny Novgorod State University, 23 Gagarin avenue, Nizhny Novgorod, \\ 603950, Russia \\ ${ }^{2}$ Nizhny Novgorod State University of Architecture and Civil Engineering, 65 Il'insky \\ street, Nizhny Novgorod, 603950, Russia
}

\begin{abstract}
In this paper we present analysis manufacturing an implanted-junction rectifier in heterostructures. We analyzed dependence of distribution of concentration of dopant in the rectifier on temperature of doping. We obtain, that increasing of temperature of doping leads to increasing of spreading of distribution of concentration of dopant and at the same time to decreasing of quantity of radiation defects. It has been shown, that variation of the temperature of heterostructure during the ion implantation gives a possibility to accelerate the technological process
\end{abstract}

\section{KEYWORDS}

Implanted-junction rectifiers, modification of profile of dopant

\section{INTRODUCTION}

In the present time they are several actual problems of solid state electronics could be considered: increasing of integration rate of integrated circuits, reliability and performance of their elements [1-10]. At the same time dimensions of elements of integrated circuits decreases. In this paper we consider a heterostructure, which includes inti itself two layers: a substrate and an epitaxial layer (see Fig. 1). We assume, that type of conductivity of the substrate ( $n$ or $p$ ) is known. The epitaxial layer has been doped by ion implantation to manufacture required type of conductivity $(p$ or $n)$. We compare two ways of implantation: (i) implantation at room temperature with future annealing of radiation defects; (ii) implantation at high temperature. We consider temperature of heating as equal to temperature of annealing of radiation defects. At the same time our aim is determination conditions, which correspond to increasing sharpness of $p$ - $n$-junctions and at the same time to increasing of homogeneity of distribution of concentration of implanted dopant in enriched area. 


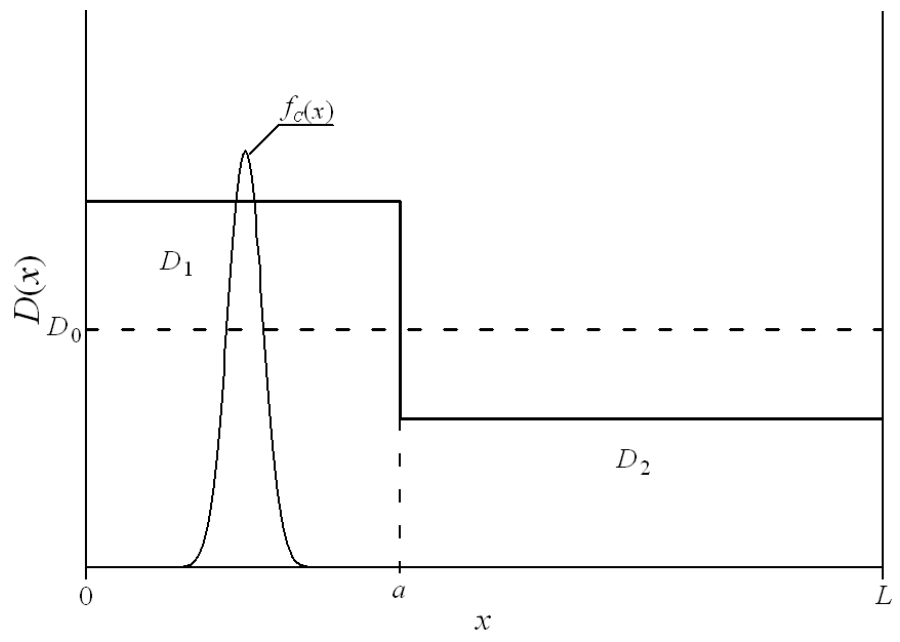

Fig.1. Heterostructure, which includes inti itself two layers: a substrate and an epitaxial layer

\section{Method Of Solution}

To analyze variation of spatio-temporal distribution of concentration of dopant with variation of temperature of ion implantation we shall solve the second Fick's law in the following form $[1,3,11]$

$$
\frac{\partial C(x, y, z, t)}{\partial t}=\frac{\partial}{\partial x}\left[D_{C} \frac{\partial C(x, y, z, t)}{\partial x}\right]+\frac{\partial}{\partial y}\left[D_{C} \frac{\partial C(x, y, z, t)}{\partial y}\right]+\frac{\partial}{\partial z}\left[D_{C} \frac{\partial C(x, y, z, t)}{\partial z}\right] .
$$

Boundary and initial conditions for the equations are

$$
\begin{aligned}
\left.\frac{\partial C(x, y, z, t)}{\partial x}\right|_{x=0} & =0,\left.\frac{\partial C(x, y, z, t)}{\partial x}\right|_{x=L_{x}}=0,\left.\frac{\partial C(x, y, z, t)}{\partial y}\right|_{y=0}=0,\left.\frac{\partial C(x, y, z, t)}{\partial y}\right|_{x=L_{y}}=0, \\
& \left.\frac{\partial C(x, y, z, t)}{\partial z}\right|_{z=0}=0,\left.\frac{\partial C(x, y, z, t)}{\partial z}\right|_{x=L_{z}}=0, C(x, y, z, 0)=f(x, y, z) .
\end{aligned}
$$

Here $C(x, y, z, t)$ is the spatio-temporal distribution of concentration of dopant. Parameter $D_{C}$ is the dopant diffusion coefficient, which depends on temperature of annealing and materials of the considered heterostructure. Spatial and temperature dependences of the dopant diffusion coefficient could be approximated by the following function [12-14]

$$
D_{C}=D_{L}(x, y, z, T)\left[1+\xi \frac{C^{\gamma}(x, y, z, t)}{P^{\gamma}(x, y, z, T)}\right]\left[1+\varsigma_{1} \frac{V(x, y, z, t)}{V^{*}}+\varsigma_{2} \frac{V^{2}(x, y, z, t)}{\left(V^{*}\right)^{2}}\right] .
$$

Here function $D_{L}(x, y, z, T)$ is the independent on any concentrations part of dopant diffusion coefficient. Function $P(x, y, z, T)$ describes the same dependences of the limit of solubility of dopant. The parameter $\gamma$ is usually equal to 1,2 or 3 . The parameter describes quantity of charged defects, which interacting (in average) with each atom of dopant. Ref.[12] describes more detailed information about dependence of dopant diffusion coefficient on concentration of dopant. Spatiotemporal distribution of concentration of radiation vacancies described by the function $V(x, y, z, t)$. 
The equilibrium distribution of concentration of vacancies has been denoted as $V^{*}$. It is known, that doping of materials by diffusion did not leads to radiation damage of materials. In this situation $\zeta_{1}=\zeta_{2}=0$. We determine spatio-temporal distributions of concentrations of radiation defects by solving the following system of equations $[13,14]$

$$
\begin{aligned}
& \frac{\partial I(x, y, z, t)}{\partial t}=\frac{\partial}{\partial x}\left[D_{I}(x, y, z, T) \frac{\partial I(x, y, z, t)}{\partial x}\right]+\frac{\partial}{\partial y}\left[D_{I}(x, y, z, T) \frac{\partial I(x, y, z, t)}{\partial y}\right]-k_{I, I}(x, y, z, T) \times \\
& \times I^{2}(x, y, z, t)+\frac{\partial}{\partial z}\left[D_{I}(x, y, z, T) \frac{\partial I(x, y, z, t)}{\partial z}\right]-k_{I, V}(x, y, z, T) I(x, y, z, t) V(x, y, z, t) \\
& \frac{\partial V(x, y, z, t)}{\partial t}=\frac{\partial}{\partial x}\left[D_{V}(x, y, z, T) \frac{\partial V(x, y, z, t)}{\partial x}\right]+\frac{\partial}{\partial y}\left[D_{V}(x, y, z, T) \frac{\partial V(x, y, z, t)}{\partial y}\right]-k_{V, V}(x, y, z, T) \times \\
& \times V^{2}(x, y, z, t)+\frac{\partial}{\partial z}\left[D_{V}(x, y, z, T) \frac{\partial V(x, y, z, t)}{\partial z}\right]-k_{I, V}(x, y, z, T) I(x, y, z, t) V(x, y, z, t) .
\end{aligned}
$$

Boundary and initial conditions for these equations are

$$
\begin{gathered}
\left.\frac{\partial \rho(x, y, z, t)}{\partial x}\right|_{x=0}=0,\left.\frac{\partial \rho(x, y, z, t)}{\partial x}\right|_{x=L_{x}}=0,\left.\frac{\partial \rho(x, y, z, t)}{\partial y}\right|_{y=0}=0,\left.\frac{\partial \rho(x, y, z, t)}{\partial y}\right|_{y=L_{y}}=0, \\
\left.\frac{\partial \rho(x, y, z, t)}{\partial z}\right|_{z=0}=0,\left.\frac{\partial \rho(x, y, z, t)}{\partial z}\right|_{z=L_{z}}=0, \rho(x, y, z, 0)=f_{\rho}(x, y, z) .
\end{gathered}
$$

Here $\rho=I, V$. We denote spatio-temperature distribution of concentration of radiation interstitials as $I(x, y, z, t) . D_{\rho}(x, y, z, T)$ are the spatio-temporal distributions of the diffusion coefficients of point radiation defects. The quadric on concentrations terms of Eqs. (4) describes generation divacancies and diinterstitials. Parameter of recombination of point radiation defects and parameters of generation of simplest complexes of point radiation defects have been denoted as the following functions $k_{I, V}(x, y, z, T), k_{I, I}(x, y, z, T)$ and $k_{V, V}(x, y, z, T)$, respectively.

Now let us calculate distributions of concentrations of divacancies $\Phi_{V}(x, y, z, t)$ and diinterstitials $\Phi_{I}(x, y, z, t)$ in space and time by solving the following system of equations $[13,14]$

$$
\begin{aligned}
& \frac{\partial \Phi_{I}(x, y, z, t)}{\partial t}=\frac{\partial}{\partial x}\left[D_{\Phi I}(x, y, z, T) \frac{\partial \Phi_{I}(x, y, z, t)}{\partial x}\right]+\frac{\partial}{\partial y}\left[D_{\Phi I}(x, y, z, T) \frac{\partial \Phi_{I}(x, y, z, t)}{\partial y}\right]+ \\
& +\frac{\partial}{\partial z}\left[D_{\Phi I}(x, y, z, T) \frac{\partial \Phi_{I}(x, y, z, t)}{\partial z}\right]+k_{I, I}(x, y, z, T) I^{2}(x, y, z, t)-k_{I}(x, y, z, T) I(x, y, z, t) \\
& \frac{\partial \Phi_{V}(x, y, z, t)}{\partial t}=\frac{\partial}{\partial x}\left[D_{\Phi V}(x, y, z, T) \frac{\partial \Phi_{V}(x, y, z, t)}{\partial x}\right]+\frac{\partial}{\partial y}\left[D_{\Phi V}(x, y, z, T) \frac{\partial \Phi_{V}(x, y, z, t)}{\partial y}\right]+ \\
& +\frac{\partial}{\partial z}\left[D_{\Phi V}(x, y, z, T) \frac{\partial \Phi_{V}(x, y, z, t)}{\partial z}\right]+k_{V, V}(x, y, z, T) V^{2}(x, y, z, t)-k_{V}(x, y, z, T) V(x, y, z, t) .
\end{aligned}
$$


Boundary and initial conditions for these equations are

$$
\begin{gathered}
\left.\frac{\partial \Phi_{\rho}(x, y, z, t)}{\partial x}\right|_{x=0}=0,\left.\frac{\partial \Phi_{\rho}(x, y, z, t)}{\partial x}\right|_{x=L_{x}}=0,\left.\frac{\partial \Phi_{\rho}(x, y, z, t)}{\partial y}\right|_{y=0}=0,\left.\frac{\partial \Phi_{\rho}(x, y, z, t)}{\partial y}\right|_{y=L_{y}}=0, \\
\left.\frac{\partial \Phi_{\rho}(x, y, z, t)}{\partial z}\right|_{z=0}=0,\left.\frac{\partial \Phi_{\rho}(x, y, z, t)}{\partial z}\right|_{z=L_{z}}=0, \Phi_{I}(x, y, z, 0)=f_{\Phi I}(x, y, z), \Phi_{V}(x, y, z, 0)=f_{\Phi V}(x, y, z) .(7)
\end{gathered}
$$

The functions $D_{\rho}(x, y, z, T)$ are the spatio-temperature distributions of the diffusion coefficients of complexes of point radiation defects. The functions $k_{I}(x, y, z, T)$ and $k_{V}(x, y, z, T)$ are the spatiotemperature distributions of parameters of decay of complexes of point radiation defects.

To determine spatio-temporal distribution of concentration of dopant we transform the Eq.(1) to the following integro-differential form

$$
\begin{aligned}
& \quad \frac{x y z}{L_{x} L_{y} L_{z}} \int_{L_{x} L_{y} L_{z}}^{x} \int_{z}^{z} C(u, v, w, t) d w d v d u=\int_{0}^{t} \int_{L_{y} L_{z}}^{y} D_{L}^{z} D_{L}(x, v, w, T)\left[1+\varsigma_{1} \frac{V(x, v, w, \tau)}{V^{*}}+\varsigma_{2} \frac{V^{2}(x, v, w, \tau)}{\left(V^{*}\right)^{2}}\right] \times \\
& \times\left[1+\xi \frac{C^{\gamma}(x, v, w, \tau)}{P^{\gamma}(x, v, w, T)}\right] \frac{\partial C(x, v, w, \tau)}{\partial x} d \tau \frac{y z}{L_{y} L_{z}}+\int_{0}^{t} \int_{L_{x}}^{x} \int_{L_{z}}^{z} D_{L}(u, y, w, T)\left[1+\xi \frac{C^{\gamma}(u, y, w, \tau)}{P^{\gamma}(x, y, z, T)}\right] \times \\
& \times\left[1+\varsigma_{1} \frac{V(u, y, w, \tau)}{V^{*}}+\varsigma_{2} \frac{V^{2}(u, y, w, \tau)}{\left(V^{*}\right)^{2}}\right] \frac{\partial C(u, y, w, \tau)}{\partial y} d \tau \frac{x z}{L_{x} L_{z}}+\int_{0}^{t} \int_{L_{x} L_{y}}^{x} \int_{L_{y}}^{y} D_{L}(u, v, z, T) \times \\
& \times\left[1+\varsigma_{1} \frac{V(u, v, z, \tau)}{V^{*}}+\varsigma_{2} \frac{V^{2}(u, v, z, \tau)}{\left(V^{*}\right)^{2}}\right]\left[1+\xi \frac{C^{\gamma}(u, v, z, \tau)}{P^{\gamma}(x, y, z, T)}\right] \frac{\partial C(u, v, z, \tau)}{\partial z} d \tau \frac{x y}{L_{x} L_{y}}+ \\
& +\frac{x y z}{L_{x} L_{y} L_{z}} \int_{L_{x}}^{x} \int_{L_{y} L_{z}}^{y} \int^{z} f(u, v, w) d w d v d u .
\end{aligned}
$$

Now let us determine solution of Eq.(1a) by Bubnov-Galerkin approach [15]. To use the approach we consider solution of the Eq.(1a) as the following series with finite number of terms $N$

$$
C_{0}(x, y, z, t)=\sum_{n=0}^{N} a_{n C} c_{n}(x) c_{n}(y) c_{n}(z) e_{n C}(t) \text {. }
$$

Here $e_{n C}(t)=\exp \left[-\pi^{2} n^{2} D_{0 c} t\left(L_{x}^{-2}+L_{y}^{-2}+L_{z}^{-2}\right)\right], c_{n}(\chi)=\cos \left(\pi n \chi / L_{\chi}\right), D_{0}$ is averaged dopant diffusion coefficient. Substitution of the series into Eq.(1a) leads to the following result

$$
\begin{aligned}
& \frac{x y z}{\pi^{2}} \sum_{n=1}^{N} \frac{a_{C}}{n^{3}} s_{n}(x) s_{n}(y) s_{n}(z) e_{n C}(t)=-\frac{y z}{L_{y} L_{z}} \int_{0}^{t} \int_{L_{y} L_{z}}^{y}\left\{\left\{1+\left[\sum_{n=1}^{N} a_{n C} c_{n}(x) c_{n}(v) c_{n}(w) e_{n C}(\tau)\right]^{\gamma} \times\right.\right. \\
& \left.\times \frac{\xi}{P^{\gamma}(x, v, w, T)}\right\}\left[1+\varsigma_{1} \frac{V(x, v, w, \tau)}{V^{*}}+\varsigma_{2} \frac{V^{2}(x, v, w, \tau)}{\left(V^{*}\right)^{2}}\right] D_{L}(x, v, w, T) \sum_{n=1}^{N} a_{n C} s_{n}(x) c_{n}(v) \times \\
& \times n c_{n}(w) e_{n C}(\tau) d \tau-\frac{x z}{L_{x} L_{z}} \int_{0}^{t} \int_{L_{x}}^{x} \iint_{L_{z}}^{z}\left\{1+\left[\sum_{m=1}^{N} a_{m C} c_{m}(u) c_{m}(y) c_{m}(w) e_{m C}(\tau)\right]^{\gamma} \frac{\xi}{P^{\gamma}(u, y, w, T)}\right\} \times \\
& \times D_{L}(u, y, w, T)\left[1+\varsigma_{1} \frac{V(u, y, w, \tau)}{V^{*}}+\varsigma_{2} \frac{V^{2}(u, y, w, \tau)}{\left(V^{*}\right)^{2}}\right]_{n=1}^{N} n c_{n}(u) s_{n}(y) c_{n}(w) e_{n C}(\tau) d \tau \times
\end{aligned}
$$




$$
\begin{gathered}
\times a_{n C}-\frac{x y}{L_{x} L_{y}} \int_{0}^{t} \int_{L_{x} L_{y}}^{x} \int_{L}^{y} D_{L}(u, v, z, T)\left\{1+\frac{\xi}{P^{\gamma}(u, v, z, T)}\left[\sum_{n=1}^{N} a_{n C} c_{n}(u) c_{n}(v) c_{n}(z) e_{n C}(\tau)\right]^{\gamma}\right\} \times \\
\times\left[1+\varsigma_{1} \frac{V(u, v, z, \tau)}{V^{*}}+\varsigma_{2} \frac{V^{2}(u, v, z, \tau)}{\left(V^{*}\right)^{2}}\right] \sum_{n=1}^{N} n a_{n C} c_{n}(u) c_{n}(v) s_{n}(z) e_{n C}(\tau) d \tau+\frac{x y z}{L_{x} L_{y} L_{z}} \times \\
\times \int_{L_{x} L_{y} L_{z}}^{x} \int_{z}^{z} f(u, v, w) d w d v d u,
\end{gathered}
$$

where $s_{n}(\chi)=\sin \left(\pi n \chi / L_{\chi}\right)$. We used condition of orthogonality to determine coefficients $a_{n}$ in the considered series. The coefficients $a_{n}$ could be calculated for any quantity of terms $N$. In the common case the relations could be written as

$$
\begin{aligned}
& -\frac{L_{x}^{2} L_{y}^{2} L_{z}^{2}}{\pi^{5}} \sum_{n=1}^{N} \frac{a_{n C}}{n^{6}} e_{n C}(t)=-\frac{L_{y} L_{z}}{2 \pi^{2}} \int_{0}^{t} \int_{0}^{L_{x} L_{y} L_{0}} \int_{0} \int_{0} D_{L}(x, y, z, T)\left\{1+\left[\sum_{n=1}^{N} a_{n C} c_{n}(x) c_{n}(y) c_{n}(z) e_{n C}(\tau)\right]^{\gamma} \times\right. \\
& \left.\times \frac{\xi}{P^{\gamma}(x, y, z, T)}\right\}\left[1+\varsigma_{1} \frac{V(x, y, z, \tau)}{V^{*}}+\varsigma_{2} \frac{V^{2}(x, y, z, \tau)}{\left(V^{*}\right)^{2}}\right] \sum_{n=1}^{N} \frac{a_{n C}}{n} s_{n}(2 x) c_{n}(y) c_{n}(z) e_{n C}(\tau) \times \\
& \times\left\{y s_{n}(y)+\frac{L_{y}}{\pi n}\left[c_{n}(y)-1\right]\right\}\left\{z s_{n}(z)+\frac{L_{z}}{\pi n}\left[c_{n}(z)-1\right]\right\} d z d y d x d \tau-\int_{0}^{t} \int_{0}^{L_{L}} \int_{0}^{L_{y} L_{z}} \int_{0} D_{L}(x, y, z, T) \times \\
& \times D_{L}(x, y, z, T)\left\{1+\left[\sum_{n=1}^{N} a_{n C} c_{n}(x) c_{n}(y) c_{n}(z) e_{n C}(\tau)\right]^{\gamma} \frac{\xi}{P^{\gamma}(x, y, z, T)}\right\}\left[1+\varsigma_{1} \frac{V(x, y, z, \tau)}{V^{*}}+\right. \\
& \left.+\varsigma_{2} \frac{V^{2}(x, y, z, \tau)}{\left(V^{*}\right)^{2}}\right]\left[1+\varsigma_{1} \frac{V(x, y, z, \tau)}{V^{*}}+\varsigma_{2} \frac{V^{2}(x, y, z, \tau)}{\left(V^{*}\right)^{2}}\right] \sum_{n=1}^{N}\left\{x s_{n}(x)+\frac{L_{x}}{\pi n}\left[c_{n}(x)-1\right]\right\} \frac{a_{n C}}{n} \times \\
& \times \frac{L_{x} L_{z}}{2 \pi^{2}} c_{n}(x) s_{n}(2 y) c_{n}(z) e_{n C}(\tau)\left\{z s_{n}(z)+\frac{L_{z}}{\pi n}\left[c_{n}(z)-1\right]\right\} d z d y d x d \tau-\frac{L_{x} L_{y}}{2 \pi^{2}} \times \\
& \times \int_{0}^{t} \int_{0}^{L_{x}} \int_{0}^{L_{y} L_{z}} \int_{0}\left\{1+\left[\sum_{n=1}^{N} a_{n C} c_{n}(x) c_{n}(y) c_{n}(z) e_{n C}(\tau)\right]^{\gamma} \frac{\xi}{P^{\gamma}(x, y, z, T)}\right\}\left[1+\varsigma_{2} \frac{V^{2}(x, y, z, \tau)}{\left(V^{*}\right)^{2}}+\right. \\
& \left.+\varsigma_{1} \frac{V(x, y, z, \tau)}{V^{*}}\right] D_{L}(x, y, z, T) \sum_{n=1}^{N} \frac{a_{n C}}{n} c_{n}(x) c_{n}(y) s_{n}(z)\left\{x s_{n}(x)+\frac{L_{x}}{\pi n}\left[c_{n}(x)-1\right]\right\} \times \\
& \times\left\{y s_{n}(y)+\frac{L_{y}}{\pi n}\left[c_{n}(y)-1\right]\right\} e_{n C}(\tau) d z d y d x d \tau+\sum_{n=1}^{N} \int_{0}^{L_{x}}\left\{x s_{n}(x)+\frac{L_{x}}{\pi n}\left[c_{n}(x)-1\right]\right\} \times \\
& \times \int_{0}^{L_{y}}\left\{y s_{n}(y)+\frac{L_{y}}{\pi n}\left[c_{n}(y)-1\right]\right] \int_{0}^{L_{z}}\left\{z s_{n}(z)+\frac{L_{z}}{\pi n}\left[c_{n}(z)-1\right]\right\} f(x, y, z) d z d y d x .
\end{aligned}
$$

As an example for $\gamma=0$ we obtain

$$
\begin{aligned}
a_{n C} & =\int_{0}^{L_{x}} \int_{0}^{L_{y}}\left\{y s_{n}(y)+\frac{L_{y}}{\pi n}\left[c_{n}(y)-1\right]\right\} \int_{0}^{L_{z}}\left\{z s_{n}(z)+\frac{L_{y}}{\pi n}\left[c_{n}(z)-1\right]\right\} f(x, y, z) d z d y\left\{x s_{n}(x)+\right. \\
& \left.\times\left[c_{n}(x)-1\right] \frac{L_{x}}{\pi n}\right\} d x\left(\frac { n } { 2 } \left\{\int_{0}^{t} \int_{0}^{L_{x}} s_{n}(2 x) \int_{0}^{L_{y}} c_{n}(y)\left\{y s_{n}(y)+\frac{L_{y}}{\pi n}\left[c_{n}(y)-1\right]\right\} \int_{0}^{L_{z}} D_{L}(x, y, z, T) \times\right.\right.
\end{aligned}
$$




$$
\begin{aligned}
& \times\left\{z s_{n}(z)+\frac{L_{y}}{\pi n}\left[c_{n}(z)-1\right]\right\}\left[1+\varsigma_{1} \frac{V(x, y, z, \tau)}{V^{*}}+\varsigma_{2} \frac{V^{2}(x, y, z, \tau)}{\left(V^{*}\right)^{2}}\right]\left[1+\frac{\xi}{P^{\gamma}(x, y, z, T)}\right] \times \\
& \times c_{n}(z) d z d y d x e_{n C}(\tau) d \tau+\int_{0}^{t} e_{n C}(\tau) \int_{0}^{L_{x}} c_{n}(x)\left\{x s_{n}(x)+\frac{L_{y}}{\pi n}\left[c_{n}(x)-1\right]\right\} \int_{0}^{L_{y}} s_{n}(2 y) \int_{0}^{L_{z}} c_{n}(z) \times \\
& \times\left\{z s_{n}(z)+\frac{L_{y}}{\pi n}\left[c_{n}(z)-1\right]\right\}\left[1+\frac{\xi}{P^{\gamma}(x, y, z, T)}\right]\left[1+\varsigma_{1} \frac{V(x, y, z, \tau)}{V^{*}}+\varsigma_{2} \frac{V^{2}(x, y, z, \tau)}{\left(V^{*}\right)^{2}}\right] \times \\
& \times D_{L}(x, y, z, T) d z d y d x d \tau+\int_{0}^{t} e_{n C}(\tau) \int_{0}^{L_{x}} c_{n}(x)\left\{x s_{n}(x)+\frac{L_{x}}{\pi n}\left[c_{n}(x)-1\right]\right\} \int_{0}^{L_{y}} c_{n}(y)\left\{s_{n}(y) \times\right. \\
& \left.\times y+\frac{L_{y}}{\pi n}\left[c_{n}(y)-1\right]\right\} \int_{0}^{\int_{z}} s_{n}(2 z) D_{L}(x, y, z, T)\left[1+\frac{\xi}{P^{\gamma}(x, y, z, T)}\right]\left[1+\varsigma_{2} \frac{V^{2}(x, y, z, \tau)}{\left(V^{*}\right)^{2}}+\right. \\
& \left.\left.\left.+\varsigma_{1} \frac{V(x, y, z, \tau)}{V^{*}}\right] d z d y d x d \tau\right\}-\frac{L_{z}^{2} L_{z}^{2} L_{z}^{2}}{\pi^{5} n^{6}} e_{n C}(t)\right)^{-1} \cdot
\end{aligned}
$$

For $\gamma=1$ one can obtain the following relation to determine required parameters

$$
a_{n C}=-\frac{\beta_{n}}{2 \alpha_{n}} \pm \sqrt{\beta_{n}^{2}+4 \alpha_{n} \int_{0}^{L_{x}} c_{n}(x) \int_{0}^{L_{y}} c_{n}(y) \int_{0}^{L_{z}} c_{n}(z) f(x, y, z) d z d y d x},
$$

where $\alpha_{n}=\frac{\xi L_{y} L_{z}}{2 \pi^{2} n} \int_{0}^{t} e_{n C}(\tau) \int_{0}^{L_{x}} s_{n}(2 x) \int_{0}^{L_{y}} c_{n}(y) \int_{0}^{L_{z}} c_{n}(z)\left[1+\varsigma_{1} \frac{V(x, y, z, \tau)}{V^{*}}+\varsigma_{2} \frac{V^{2}(x, y, z, \tau)}{\left(V^{*}\right)^{2}}\right] \times$ $\times \frac{D_{L}(x, y, z, T)}{P(x, y, z, T)}\left\{y s_{n}(y)+\frac{L_{y}}{\pi n}\left[c_{n}(y)-1\right]\right\}\left\{z s_{n}(z)+\frac{L_{z}}{\pi n}\left[c_{n}(z)-1\right]\right\} d z d y d x d \tau+\frac{\xi L_{x} L_{z}}{2 \pi^{2} n} \times$ $\times \int_{0}^{t} e_{n C}(\tau) \int_{0}^{L_{x}} c_{n}(x)\left\{x s_{n}(x)+\frac{L_{x}}{\pi n}\left[c_{n}(x)-1\right]\right\} \int_{0}^{L_{y}} \int_{0}^{L_{z}} c_{n}(z) \frac{D_{L}(x, y, z, T)}{P(x, y, z, T)}\left\{z s_{n}(z)-\frac{L_{z}}{\pi n}\left[c_{n}(z)-1\right]\right\} \times$ $\times \frac{D_{L}(x, y, z, T)}{P(x, y, z, T)}\left[1+\varsigma_{1} \frac{V(x, y, z, \tau)}{V^{*}}+\varsigma_{2} \frac{V^{2}(x, y, z, \tau)}{\left(V^{*}\right)^{2}}\right] d z s_{n}(2 y) d y d x d \tau+\frac{\xi L_{x} L_{y}}{2 \pi^{2} n} \times$ $\times \int_{0}^{t} e_{n C}(\tau) \int_{0}^{L_{x}} c_{n}(x) \int_{0}^{L_{y}} c_{n}(y) \int_{0}^{L_{z}} s_{n}(2 z) \frac{D_{L}(x, y, z, T)}{P(x, y, z, T)}\left[1+\varsigma_{1} \frac{V(x, y, z, \tau)}{V^{*}}+\varsigma_{2} \frac{V^{2}(x, y, z, \tau)}{\left(V^{*}\right)^{2}}\right] \times$ $\times\left\{x s_{n}(x)+\frac{L_{x}}{\pi n}\left[c_{n}(x)-1\right]\right\}\left\{y s_{n}(y)+\frac{L_{y}}{\pi n}\left[c_{n}(y)-1\right]\right\} d z d y d x d \tau, \beta_{n}=\frac{L_{y} L_{z}}{2 n \pi^{2}} \int_{0}^{t} e_{n C}(\tau) \times$ $\times \int_{0}^{L_{x}} S_{n}(2 x) \int_{0}^{L_{y}} c_{n}(y)\left\{y s_{n}(y)+\frac{L_{y}}{\pi n}\left[c_{n}(y)-1\right]\right\} \int_{0}^{L_{z}} c_{n}(z)\left[1+\varsigma_{1} \frac{V(x, y, z, \tau)}{V^{*}}+\varsigma_{2} \frac{V^{2}(x, y, z, \tau)}{\left(V^{*}\right)^{2}}\right] \times$ $\times D_{L}(x, y, z, T)\left\{z s_{n}(z)+\frac{L_{z}}{\pi n}\left[c_{n}(z)-1\right]\right\} d z d y d x d \tau+\frac{L_{x} L_{z}}{2 n \pi^{2}} \int_{0}^{t} e_{n C}(\tau) \int_{0}^{L_{x}} c_{n}(x) \int_{0}^{L_{y}} s_{n}(2 y) \times$ 


$$
\begin{aligned}
& \times\left\{x s_{n}(x)+\frac{L_{x}}{\pi n}\left[c_{n}(x)-1\right]\right\} \int_{0}^{L_{z}} D_{L}(x, y, z, T) c_{n}(z)\left[1+\varsigma_{1} \frac{V(x, y, z, \tau)}{V^{*}}+\varsigma_{2} \frac{V^{2}(x, y, z, \tau)}{\left(V^{*}\right)^{2}}\right] \times \\
& \times\left\{z s_{n}(z)+\frac{L_{z}}{\pi n}\left[c_{n}(z)-1\right]\right\} d z d y d x d \tau+\frac{L_{x} L_{y}}{2 n \pi^{2}} \int_{0}^{t} e_{n C}(\tau) \int_{0}^{L_{x}}\left\{x s_{n}(x)+\frac{L_{x}}{\pi n}\left[c_{n}(x)-1\right]\right\} \times \\
& \times c_{n}(x) \int_{0}^{L_{y}}\left\{y s_{n}(y)+\frac{L_{y}}{\pi n}\left[c_{n}(y)-1\right]\right\} \int_{0}^{L_{z}} D_{L}(x, y, z, T)\left[1+\varsigma_{1} \frac{V(x, y, z, \tau)}{V^{*}}+\varsigma_{2} \frac{V^{2}(x, y, z, \tau)}{\left(V^{*}\right)^{2}}\right] \times \\
& \times s_{n}(2 z) d z c_{n}(y) d y d x d \tau-L_{x}^{2} L_{y}^{2} L_{z}^{2} e_{n C}(t) / \pi^{5} n^{6} .
\end{aligned}
$$

The same approach could be used for calculation parameters $a_{n}$ for different values of parameter $\gamma$. However the relations are bulky and will not be presented in the paper. Advantage of the approach is absent of necessity to join dopant concentration on interfaces of heterostructure.

The same Bubnov-Galerkin approach has been used for solution the Eqs.(4). Previously we transform the differential equations to the following integro- differential form

$$
\begin{aligned}
& \frac{x y z}{L_{x} L_{y} L_{z}} \int_{L_{x} L_{y} L_{z}}^{x} \int_{z}^{z} I(u, v, w, t) d w d v d u=\frac{y z}{L_{y} L_{z}} \int_{0}^{t} \int_{L_{y} L_{z}}^{y} \int_{I}^{z} D_{I}(x, v, w, T) \frac{\partial I(x, v, w, \tau)}{\partial x} d w d v d \tau+ \\
& +\frac{x z}{L_{x} L_{z}} \int_{0}^{t} \int_{L_{x} L_{z}}^{x} D_{I}^{z}(u, y, w, T) \frac{\partial I(u, y, w, \tau)}{\partial x} d w d u d \tau-\frac{x y z}{L_{x} L_{y} L_{z}} \int_{L_{x} L_{y} L_{z}}^{x} \int_{I, V}^{z} k_{I, V}(u, v, w, T) I(u, v, w, t) \times \\
& \times V(u, v, w, t) d w d v d u+\frac{x y}{L_{x} L_{y}} \int_{0}^{t} \int_{L_{x} L_{y}}^{x} \frac{\partial I(u, v, z, \tau)}{\partial z} D_{I}(u, v, z, T) d v d u d \tau-\frac{x y z}{L_{x} L_{y} L_{z}} \times \\
& \times \int_{L_{x}}^{x} \int_{L_{y} L_{z}}^{y} \int_{I, I}^{z} k_{I}(u, v, w, T) I^{2}(u, v, w, t) d w d v d u+\frac{x y z}{L_{x} L_{y} L_{z}} \int_{L_{x}}^{x} \int_{L_{y}}^{y} \int_{L_{z}}^{z} f_{I}(u, v, w) d w d v d u \\
& \frac{x y z}{L_{x} L_{y} L_{z}} \int_{L_{x}}^{x} \int_{L_{y} L_{z}}^{y} z(u, v, w, t) d w d v d u=\frac{y z}{L_{y} L_{z}} \int_{0}^{t} \int_{L_{y} L_{z}}^{y} \int_{L_{z}}^{z} D_{V}(x, v, w, T) \frac{\partial V(x, v, w, \tau)}{\partial x} d w d v d \tau+ \\
& +\frac{x z}{L_{x} L_{z}} \int_{0}^{t} \int_{L_{x} L_{z}}^{x} \int_{V}^{z} D_{V}(u, y, w, T) \frac{\partial V(u, y, w, \tau)}{\partial x} d w d u d \tau+\frac{x y}{L_{x} L_{y}} \int_{0}^{t} \int_{L_{x} L_{y}}^{x} \int_{\frac{\partial}{\partial}}^{\partial V(u, v, z, \tau)} \frac{}{\partial z} \times
\end{aligned}
$$

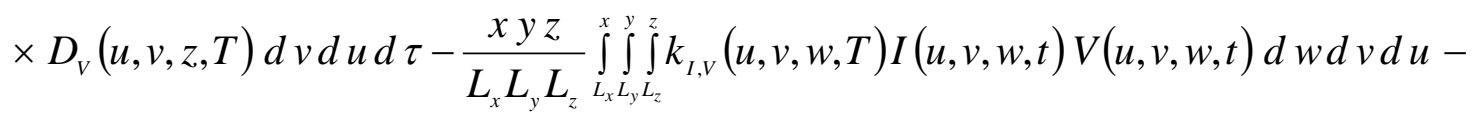

$$
\begin{aligned}
& -\frac{x y z}{L_{x} L_{y} L_{z}} \int_{L_{x} L_{y} L_{z}}^{x} \int_{V}^{z} k_{V, V}(u, v, w, T) V^{2}(u, v, w, t) d w d v d u+\frac{x y z}{L_{x} L_{y} L_{z}} \int_{L_{x} L_{y} L_{z}}^{x} \int_{V}^{z} f_{V}(u, v, w) d w d v d u .
\end{aligned}
$$

Spatio-temporal distributions of concentrations of point defects have been determined as the following series

$$
\rho_{0}(x, y, z, t)=\sum_{n=1}^{N} a_{n \rho} c_{n}(x) c_{n}(y) c_{n}(z) e_{n \rho}(t)
$$

Parameters $a_{n \rho}$ should be determined in future. Substitution of the series into Eqs.(4a) leads to the following results 


$$
\begin{aligned}
& \frac{x y z}{\pi^{3}} \sum_{n=1}^{N} \frac{a_{n I}}{n^{3}} s_{n}(x) s_{n}(y) s_{n}(z) e_{n I}(t)=-\frac{y z \pi}{L_{x} L_{y} L_{z}} \sum_{n=1}^{N} a_{n I} \int_{0}^{t} \int_{L_{y}}^{y} c_{n}(y) \int_{L_{z}}^{z} c_{n}(z) D_{I}(x, v, w, T) d w d v \times \\
& \times e_{n I}(\tau) d \tau s_{n}(x)-\frac{x z \pi}{L_{x} L_{y} L_{z}} \sum_{n=1}^{N} a_{n I} s_{n}(y) \int_{0}^{t} e_{n I}(\tau) \int_{L_{x}}^{x} c_{n}(x) \int_{L_{z}}^{z} c_{n}(z) D_{I}(u, y, w, T) d w d u d \tau- \\
& -\frac{x y \pi}{L_{x} L_{y} L_{z}} \sum_{n=1}^{N} a_{n I} s_{n}(z) \int_{0}^{t} e_{n I}(\tau) \int_{L_{x}}^{x} c_{n}(x) \int_{L_{y}}^{y} c_{n}(y) D_{I}(u, v, z, T) d v d u d \tau-\int_{L_{x}}^{x} \int_{y}^{y} \int_{L_{z}}^{z} k_{I I I}(u, v, v, T) \times \\
& \times\left[\sum_{n=1}^{N} a_{n I} c_{n}(u) c_{n}(v) c_{n}(w) e_{n I}(t)\right]^{2} d w d v d u \frac{x y z}{L_{x} L_{y} L_{z}}-\frac{x y z}{L_{x} L_{y} L_{z}} \int_{L_{x} L_{y} L_{z}}^{x} \int_{n=1}^{z} \sum_{n I}^{N} a_{n I} c_{n}(u) c_{n}(v) c_{n}(w) \times \\
& \times e_{n I}(t) \sum_{n=1}^{N} a_{n V} c_{n}(u) c_{n}(v) c_{n}(w) e_{n V}(t) k_{I, V}(u, v, v, T) d w d v d u+\int_{L_{x} L_{y} L_{2}}^{x} \int_{I}^{z} f_{I}(u, v, w) d w d v d u \times \\
& \times x y z / L_{x} L_{y} L_{z} \\
& \frac{x y z}{\pi^{3}} \sum_{n=1}^{N} \frac{a_{n V}}{n^{3}} s_{n}(x) s_{n}(y) s_{n}(z) e_{n V}(t)=-\frac{y z \pi}{L_{x} L_{y} L_{z}} \sum_{n=1}^{N} a_{n V} \int_{0}^{t} \int_{L_{y}}^{y} c_{n}(y) \int_{L_{z}}^{z} c_{n}(z) D_{V}(x, v, w, T) d w d v \times \\
& \times e_{n V}(\tau) d \tau s_{n}(x)-\frac{x z \pi}{L_{x} L_{y} L_{z}} \sum_{n=1}^{N} a_{n V} s_{n}(y) \int_{0}^{t} e_{n V}(\tau) \int_{L_{x}}^{x} c_{n}(x) \int_{L_{z}}^{z} c_{n}(z) D_{V}(u, y, w, T) d w d u d \tau- \\
& -\frac{x y \pi}{L_{x} L_{y} L_{z}} \sum_{n=1}^{N} a_{n V} s_{n}(z) \int_{0}^{t} e_{n V}(\tau) \int_{L_{x}}^{x} c_{n}(x) \int_{L_{y}}^{y} c_{n}(y) D_{V}(u, v, z, T) d v d u d \tau-\int_{L_{x}}^{x} \int_{L_{y} L_{z}}^{y} \int_{V, V}(u, v, v, T) \times \\
& \times\left[\sum_{n=1}^{N} a_{n V} c_{n}(u) c_{n}(v) c_{n}(w) e_{n I}(t)\right]^{2} d w d v d u \frac{x y z}{L_{x} L_{y} L_{z}}-\frac{x y z}{L_{x} L_{y} L_{z}} \int_{L_{x} L_{y} L_{z}}^{x} \int_{n=1}^{z} \sum_{n I}^{N} a_{n I} c_{n}(u) c_{n}(v) c_{n}(w) \times \\
& \times e_{n I}(t) \sum_{n=1}^{N} a_{n V} c_{n}(u) c_{n}(v) c_{n}(w) e_{n V}(t) k_{I, V}(u, v, v, T) d w d v d u+\int_{L_{x} L_{y} L_{2}}^{x} \int_{V}^{z} f_{V}(u, v, w) d w d v d u \times \\
& \times x y z / L_{x} L_{y} L_{z} .
\end{aligned}
$$

We used orthogonality condition of functions of the considered series framework the heterostructure to calculate coefficients $a_{n \rho}$. The coefficients $a_{n}$ could be calculated for any quantity of terms $N$. In the common case equations for the required coefficients could be written as

$$
\begin{aligned}
& -\frac{L_{x}^{2} L_{y}^{2} L_{z}^{2}}{\pi^{5}} \sum_{n=1}^{N} \frac{a_{n I}}{n^{6}} e_{n I}(t)=-\frac{1}{2 \pi L_{x}} \sum_{n=1}^{N} \frac{a_{n I}}{n^{2}} \int_{0}^{t} \int_{0}^{L_{x}}\left[1-c_{n}(2 x)\right] \int_{0}^{L_{y}}\left\{L_{y}+y s_{n}(2 y)+\frac{L_{y}}{2 \pi n}\left[c_{n}(2 y)-1\right]\right\} \times \\
& \times \int_{0}^{L_{z}} D_{I}(x, y, z, T)\left\{z s_{n}(z)+\frac{L_{z}}{2 \pi n}\left[c_{n}(z)-1\right]\right\} d z d y d x e_{n I}(\tau) d \tau-\frac{1}{2 \pi L_{y}} \sum_{n=1}^{N} \frac{a_{n I}}{n^{2}} \int_{0}^{t} \int_{0}^{L_{x}}\left\{x s_{n}(2 x)+\right. \\
& \left.+L_{x}+\frac{L_{x}}{\pi n}\left[c_{n}(2 x)-1\right]\right\} \int_{0}^{L_{y} L_{z}} \int_{0} D_{I}(x, y, z, T)\left\{L_{z}+z s_{n}(2 z)+\frac{L_{z}}{2 \pi n}\left[c_{n}(2 z)-1\right]\right\} d z\left[1-c_{n}(2 y)\right] \times \\
& \times d y d x e_{n I}(\tau) d \tau \int_{0}^{L_{z}} D_{I}(x, y, z, T)\left\{L_{z}+z s_{n}(2 z)+\frac{L_{z}}{2 \pi n}\left[c_{n}(2 z)-1\right]\right\} d z d y d x e_{n I}(\tau) d \tau- \\
& -\frac{1}{2 \pi L_{z}} \sum_{n=1}^{N} \frac{a_{n I}}{n^{2}} \int_{0}^{t} \int_{0}^{L_{x}}\left\{L_{x}+x s_{n}(2 x)+\frac{L_{x}}{2 \pi n}\left[c_{n}(2 x)-1\right]\right\} \int_{0}^{L_{y}}\left\{L_{y}+y s_{n}(2 y)+\frac{L_{y}}{2 \pi n}\left[c_{n}(2 y)-1\right]\right\} \times
\end{aligned}
$$




$$
\begin{aligned}
& \times \int_{0}^{L_{z}}\left[1-c_{n}(2 z)\right] D_{I}(x, y, z, T) d z d y d x e_{n I}(\tau) d \tau-\sum_{n=1}^{N} a_{n I}^{2} e_{n I}(2 t) \int_{0}^{L_{x}}\left\{L_{x}+\frac{L_{x}}{2 \pi n}\left[c_{n}(2 x)-1\right]+\right. \\
& \left.+x s_{n}(2 x)\right\} \int_{0}^{L_{y}}\left\{L_{y}+y s_{n}(2 y)+\frac{L_{y}}{2 \pi n}\left[c_{n}(2 y)-1\right]\right\} \int_{0}^{L_{z}} k_{I, I}(x, y, z, T)\left\{L_{z}+\frac{L_{z}}{2 \pi n}\left[c_{n}(2 z)-1\right]+\right. \\
& \left.+z s_{n}(2 z)\right\} d z d y d x-\sum_{n=1}^{N} a_{n I} a_{n V} e_{n I}(t) e_{n V}(t) \int_{0}^{L_{x}}\left\{L_{x}+x s_{n}(2 x)+\frac{L_{x}}{2 \pi n}\left[c_{n}(2 x)-1\right]\right\} \int_{0}^{L_{y}}\left\{L_{y}+\right. \\
& +y s_{n}(2 y)+\frac{L_{y}}{2 \pi n}\left[c_{n}(2 y)-1\right] \int_{0}^{L_{z}} k_{I, V}(x, y, z, T)\left\{L_{z}+z s_{n}(2 z)+\frac{L_{z}}{2 \pi n}\left[c_{n}(2 z)-1\right]\right\} d z \times \\
& \times d y d x+\sum_{n=1}^{N} \int_{0}^{L_{x}}\left\{x s_{n}(x)+\frac{L_{x}}{\pi n}\left[c_{n}(x)-1\right]\right\} \int_{0}^{L_{y}}\left\{y s_{n}(y)+\frac{L_{y}}{\pi n}\left[c_{n}(y)-1\right]\right\} \int_{0}^{L_{z}} f_{I}(x, y, z, T) \times \\
& \times\left\{L_{z}+z s_{n}(2 z)+\frac{L_{z}}{2 \pi n}\left[c_{n}(2 z)-1\right]\right\} d z d y d x \\
& -\frac{L_{x}^{2} L_{y}^{2} L_{z}^{2}}{\pi^{5}} \sum_{n=1}^{N} \frac{a_{n V}}{n^{6}} e_{n V}(t)=-\frac{1}{2 \pi L_{x}} \sum_{n=1}^{N} \frac{a_{n V}}{n^{2}} \int_{0}^{t} \int_{0}^{L_{x}}\left[1-c_{n}(2 x)\right] \int_{0}^{L_{y}}\left\{L_{y}+y s_{n}(2 y)+\frac{L_{y}}{2 \pi n}\left[c_{n}(2 y)-1\right]\right\} \times
\end{aligned}
$$$$
\times \int_{0}^{L_{z}} D_{V}(x, y, z, T)\left\{z s_{n}(z)+\frac{L_{z}}{2 \pi n}\left[c_{n}(z)-1\right]\right\} d z d y d x e_{n V}(\tau) d \tau-\frac{1}{2 \pi L_{y}} \sum_{n=1}^{N} \frac{a_{n V}}{n^{2}} \int_{0}^{t} \int_{0}^{L_{x}}\left\{x s_{n}(2 x)+\right.
$$$$
\left.+L_{x}+\frac{L_{x}}{\pi n}\left[c_{n}(2 x)-1\right]\right\} \int_{0}^{L_{y} L_{z}} \int_{0} D_{V}(x, y, z, T)\left\{L_{z}+z s_{n}(2 z)+\frac{L_{z}}{2 \pi n}\left[c_{n}(2 z)-1\right]\right\} d z\left[1-c_{n}(2 y)\right] \times
$$
$\times d y d x e_{n V}(\tau) d \tau \int_{0}^{L_{z}} D_{V}(x, y, z, T)\left\{L_{z}+z s_{n}(2 z)+\frac{L_{z}}{2 \pi n}\left[c_{n}(2 z)-1\right]\right\} d z d y d x e_{n V}(\tau) d \tau-$ $-\frac{1}{2 \pi L_{z}} \sum_{n=1}^{N} \frac{a_{n V}}{n^{2}} \int_{0}^{t} \int_{0}^{L_{x}}\left\{L_{x}+x s_{n}(2 x)+\frac{L_{x}}{2 \pi n}\left[c_{n}(2 x)-1\right]\right\} \int_{0}^{L_{y}}\left\{L_{y}+y s_{n}(2 y)+\frac{L_{y}}{2 \pi n}\left[c_{n}(2 y)-1\right]\right\} \times$ $\times \int_{0}^{L_{2}}\left[1-c_{n}(2 z)\right] D_{V}(x, y, z, T) d z d y d x e_{n V}(\tau) d \tau-\sum_{n=1}^{N} a_{n V}^{2} e_{n V}(2 t) \int_{0}^{L_{x}}\left\{L_{x}+\frac{L_{x}}{2 \pi n}\left[c_{n}(2 x)-1\right]+\right.$ $\left.+x s_{n}(2 x)\right\} \int_{0}^{L_{y}}\left\{L_{y}+y s_{n}(2 y)+\frac{L_{y}}{2 \pi n}\left[c_{n}(2 y)-1\right]\right\} \int_{0}^{L_{z}} k_{V, V}(x, y, z, T)\left\{L_{z}+\frac{L_{z}}{2 \pi n}\left[c_{n}(2 z)-1\right]+\right.$ $\left.+z s_{n}(2 z)\right\} d z d y d x-\sum_{n=1}^{N} a_{n I} a_{n V} e_{n I}(t) e_{n V}(t) \int_{0}^{L_{x}}\left\{L_{x}+x s_{n}(2 x)+\frac{L_{x}}{2 \pi n}\left[c_{n}(2 x)-1\right]\right\} \int_{0}^{L_{y}}\left\{L_{y}+\right.$ $\left.+y s_{n}(2 y)+\frac{L_{y}}{2 \pi n}\left[c_{n}(2 y)-1\right]\right\} \int_{0}^{L_{z}} k_{I, V}(x, y, z, T)\left\{L_{z}+z s_{n}(2 z)+\frac{L_{z}}{2 \pi n}\left[c_{n}(2 z)-1\right]\right\} d z \times$ $\times d y d x+\sum_{n=1}^{N} \int_{0}^{L_{x}}\left\{x s_{n}(x)+\frac{L_{x}}{\pi n}\left[c_{n}(x)-1\right]\right\} \int_{0}^{L_{y}}\left\{y s_{n}(y)+\frac{L_{y}}{\pi n}\left[c_{n}(y)-1\right]\right\} \int_{0}^{L_{z}} f_{V}(x, y, z, T) \times$

$$
\times\left\{L_{z}+z s_{n}(2 z)+\frac{L_{z}}{2 \pi n}\left[c_{n}(2 z)-1\right]\right\} d z d y d x .
$$


In the final form relations for required parameters could be written as

$$
a_{n I}=-\frac{b_{3}+A}{4 b_{4}} \pm \sqrt{\frac{\left(b_{3}+A\right)^{2}}{4}-4 b_{4}\left(y+\frac{b_{3} y-\gamma_{n V} \lambda_{n I}^{2}}{A}\right)}, a_{n V}=-\frac{\gamma_{n l} a_{n I}^{2}+\delta_{n I} a_{n I}+\lambda_{n I}}{\chi_{n I} a_{n I}},
$$

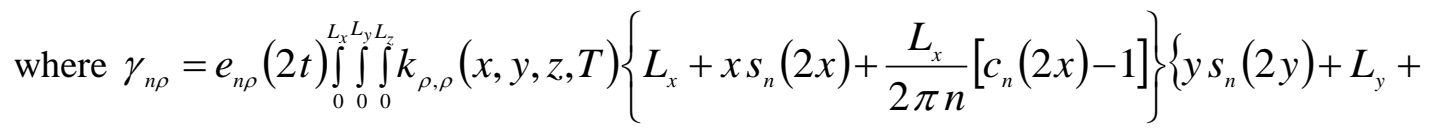
$\left.+\frac{L_{y}}{2 \pi n}\left[c_{n}(2 y)-1\right]\right\}\left\{L_{z}+z s_{n}(2 z)+\frac{L_{z}}{2 \pi n}\left[c_{n}(2 z)-1\right]\right\} d z d y d x, \delta_{n \rho}=\frac{1}{2 \pi L_{x} n^{2}} \int_{0}^{t} e_{n \rho}(\tau) \times$ $\times \int_{0}^{L_{L} L_{y}}\left\{y s_{n}(y)+\frac{L_{y}}{2 \pi n}\left[c_{n}(y)-1\right]\right\} \int_{0}^{L_{z}}\left\{z s_{n}(z)+\frac{L_{z}}{2 \pi n}\left[c_{n}(z)-1\right]\right\} D_{\rho}(x, y, z, T) d z d y[1-$ $\left.-c_{n}(2 x)\right] d x d \tau+\frac{1}{2 \pi L_{y} n^{2}} \int_{0}^{t} e_{n \rho}(\tau) \int_{0}^{L_{x}}\left\{L_{x}+x s_{n}(2 x)+\frac{L_{x}}{\pi n}\left[c_{n}(2 x)-1\right]\right\} \int_{0}^{L_{y}}\left[1-c_{n}(2 y)\right] \int_{0}^{L_{z}}\left\{L_{z}+\right.$ $\left.+z s_{n}(2 z)+\frac{L_{z}}{2 \pi n}\left[c_{n}(2 z)-1\right]\right\} D_{\rho}(x, y, z, T) d z d y d x d \tau+\frac{1}{2 \pi L_{z} n^{2}} \int_{0}^{t} e_{n \rho}(\tau) \int_{0}^{L_{x}}\left\{x s_{n}(2 x)+\right.$ $\left.+L_{x}+\frac{L_{x}}{\pi n}\left[c_{n}(2 x)-1\right]\right\} \int_{0}^{L_{y}}\left\{L_{y}+y s_{n}(y)+\frac{L_{y}}{2 \pi n}\left[c_{n}(y)-1\right]\right\} \int_{0}^{L_{z}}\left[1-c_{n}(2 z)\right] D_{\rho}(x, y, z, T) d z \times$ $\times d y d x d \tau-\frac{L_{x}^{2} L_{y}^{2} L_{z}^{2}}{\pi^{5} n^{6}} e_{n \rho}(t), \chi_{n I V}=\int_{0}^{L_{x}}\left\{x s_{n}(x)+\frac{L_{x}}{\pi n}\left[c_{n}(x)-1\right]\right\} \int_{0}^{L_{y}}\left\{L_{y}+\frac{L_{y}}{2 \pi n}\left[c_{n}(2 y)-1\right]+\right.$ $\left.+y s_{n}(2 y)\right\} \int_{0}^{L_{z}} k_{I, V}(x, y, z, T)\left\{L_{z}+z s_{n}(2 z)+\frac{L_{z}}{2 \pi n}\left[c_{n}(2 z)-1\right]\right\} d z d y d x e_{n I}(t) e_{n V}(t)$, $\lambda_{n \rho}=\int_{0}^{L_{x}}\left\{x s_{n}(x)+\frac{L_{x}}{\pi n}\left[c_{n}(x)-1\right]\right\} \int_{0}^{L_{y}}\left\{y s_{n}(y)+\frac{L_{y}}{\pi n}\left[c_{n}(y)-1\right]\right\} \int_{0}^{L_{z}}\left\{z s_{n}(z)+\frac{L_{z}}{\pi n}\left[c_{n}(z)-1\right]\right\} \times$ $\times f_{\rho}(x, y, z, T) d z d y d x, b_{4}=\gamma_{n V} \gamma_{n I}^{2}-\gamma_{n I} \chi_{n I}^{2}, b_{3}=2 \gamma_{n V} \gamma_{n I} \delta_{n I}-\delta_{n I} \chi_{n I}^{2}-\delta_{n V} \chi_{n l} \gamma_{n I}$, $A=\sqrt{8 y+b_{3}^{2}-4 b_{2}}, b_{2}=\gamma_{n V} \delta_{n I}^{2}+2 \lambda_{n I} \gamma_{n V} \gamma_{n I}-\delta_{n V} \chi_{n I} \delta_{n I}+\left(\lambda_{n V}-\lambda_{n I}\right) \chi_{n I}^{2}, b_{1}=2 \lambda_{n I} \times$ $\times \gamma_{n V} \delta_{n I}-\delta_{n V} \chi_{n I} \lambda_{n I}, y=\sqrt[3]{\sqrt{q^{2}+p^{3}}-q}-\sqrt[3]{\sqrt{q^{2}+p^{3}}+q}-\frac{b_{3}}{3 b_{4}}, p=\frac{3 b_{2} b_{4}-b_{3}^{2}}{9 b_{4}^{2}}$, $q=\left(2 b_{3}^{3}-9 b_{2} b_{3}+27 b_{1} b_{4}^{2}\right) / 54 b_{4}^{3}$.

We determine distributions of concentrations of simplest complexes of radiation defects in space and time as the following functional series

$$
\Phi_{\rho 0}(x, y, z, t)=\sum_{n=1}^{N} a_{n \Phi \rho} c_{n}(x) c_{n}(y) c_{n}(z) e_{n \rho}(t) .
$$

Here $a_{n \Phi_{\rho}}$ are the coefficients, which should be determined. Let us previously transform the Eqs. (6) to the following integro-differential form

$$
\frac{x y z}{L_{x} L_{y} L_{z}} \int_{L_{x} L_{y} L_{z}}^{x} \int_{L_{z}}^{z} \Phi_{I}(u, v, w, t) d w d v d u=\int_{0}^{t} \int_{L_{y} L_{z}}^{y} \int_{L_{I}}^{z} D_{0}(x, v, w, T) \frac{\partial \Phi_{I}(x, v, w, \tau)}{\partial x} d w d v d \tau \times
$$


International Journal of Applied Control, Electrical and Electronics Engineering (IJACEEE) Vol 4, No. 1, February 2016

$$
\begin{aligned}
& \times \frac{y z}{L_{y} L_{z}}+\frac{x z}{L_{x} L_{z}} \int_{0}^{t} \int_{0}^{x} \int_{L_{x} L_{z}}^{z} D_{\Phi I}(u, y, w, T) \frac{\partial \Phi_{I}(u, y, w, \tau)}{\partial y} d w d u d \tau+\frac{x y}{L_{x} L_{y}} \int_{0}^{t} \int_{L_{x}}^{x} \int_{L_{y}}^{y} D_{\Phi I}(u, v, z, T) \times \\
& \times \frac{\partial \Phi_{I}(u, v, z, \tau)}{\partial z} d v d u d \tau+\frac{x y z}{L_{x} L_{y} L_{z}} \int_{L_{x} L_{y} L_{L_{z}}}^{y} \int_{I, I}^{z} k_{I}(u, v, w, T) I^{2}(u, v, w, \tau) d w d v d u-(6 a)
\end{aligned}
$$

$-\frac{x y z}{L_{x} L_{y} L_{z}} \int_{L_{x} L_{y} L_{z}}^{x} \int_{I}^{z} k_{I}(u, v, w, T) I(u, v, w, \tau) d w d v d u+\frac{x y z}{L_{x} L_{y} L_{z}} \int_{L_{x} L_{y} L_{z}}^{x} \int^{z} f_{\Phi I}(u, v, w) d w d v d u$ $\frac{x y z}{L_{x} L_{y} L_{z}} \int_{L_{x} L_{y} L_{z}}^{x} \int_{V}^{z} \Phi_{V}(u, v, w, t) d w d v d u=\int_{0}^{t} \int_{L_{y} L_{z}}^{y} \int_{\Phi V}^{z} D_{0}(x, v, w, T) \frac{\partial \Phi_{V}(x, v, w, \tau)}{\partial x} d w d v d \tau \times$ $\times \frac{y z}{L_{y} L_{z}}+\frac{x z}{L_{x} L_{z}} \int_{0}^{t} \int_{L_{x} L_{z}}^{x} D_{\Phi V}(u, y, w, T) \frac{\partial \Phi_{V}(u, y, w, \tau)}{\partial y} d w d u d \tau+\frac{x y}{L_{x} L_{y}} \int_{0}^{t} \int_{L_{x} L_{y}}^{x} \int_{\Phi V}^{y} D_{\Phi}(u, v, z, T) \times$ $\times \frac{\partial \Phi_{V}(u, v, z, \tau)}{\partial z} d v d u d \tau+\frac{x y z}{L_{x} L_{y} L_{z}} \int_{L_{x} L_{y} L_{z}}^{x} \int_{V, V}^{z} k_{V}(u, v, w, T) V^{2}(u, v, w, \tau) d w d v d u-$ $-\frac{x y z}{L_{x} L_{y} L_{z}} \int_{L_{x} L_{y} L_{z}}^{x} \int_{V}^{z} k_{V}(u, v, w, T) V(u, v, w, \tau) d w d v d u+\frac{x y z}{L_{x} L_{y} L_{z}} \int_{L_{x} L_{y} L_{z}}^{x} \int_{\Phi V}^{z} f_{\Phi}(u, v, w) d w d v d u$.

Substitution of the previously considered series in the Eqs.(6a) leads to the following form

$$
\begin{aligned}
& -x y z \sum_{n=1}^{N} \frac{a_{n \Phi I}}{\pi^{3} n^{3}} s_{n}(x) s_{n}(y) s_{n}(z) e_{n I}(t)=-\frac{y z \pi}{L_{x} L_{y} L_{z}} \sum_{n=1}^{N} n a_{n \Phi I} s_{n}(x) e_{n I}(t) \int_{0}^{t} \int_{L_{y} L_{z}}^{y} \int_{n}^{z} c_{n}(v) c_{n}(w) \times \\
& \times D_{\Phi I}(x, v, w, T) d w d v d \tau-\frac{x z \pi}{L_{x} L_{y} L_{z}} \sum_{n=1}^{N} a_{n \Phi I} \int_{0}^{t} \int_{L_{x}}^{x} \int_{z}^{z} c_{n}(u) c_{n}(w) D_{\Phi I}(u, v, w, T) d w d u d \tau \times \\
& \times n s_{n}(y) e_{n \Phi I}(t)-\frac{x y \pi}{L_{x} L_{y} L_{z}} \sum_{n=1}^{N} n a_{n \Phi I} s_{n}(z) e_{n \Phi I}(t) \int_{0}^{t} \int_{L_{x} L_{y}}^{x} \int_{n}^{y} c_{n}(u) c_{n}(v) D_{\Phi I}(u, v, z, T) d v d u d \tau+ \\
& +\frac{x y z}{L_{x} L_{y} L_{z}} \int_{L_{x} L_{y} L_{z}}^{x} \int_{I, I}^{z} k_{1}(u, v, w, T) I^{2}(u, v, w, \tau) d w d v d u+\int_{L_{x} L_{y} L_{z}}^{x} \int_{\Phi I}^{z} f_{\Phi}(u, v, w) d w d v d u \times \\
& \times \frac{x y z}{L_{x} L_{y} L_{z}}-\frac{x y z}{L_{x} L_{y} L_{z}} \int_{L_{x}}^{x} \int_{L_{y} L_{z}}^{y} \int_{I}^{z} k_{I}(u, v, w, T) I(u, v, w, \tau) d w d v d u \\
& -x y z \sum_{n=1}^{N} \frac{a_{n \Phi V}}{\pi^{3} n^{3}} s_{n}(x) s_{n}(y) s_{n}(z) e_{n V}(t)=-\frac{y z \pi}{L_{x} L_{y} L_{z}} \sum_{n=1}^{N} n a_{n \Phi V} s_{n}(x) e_{n V}(t) \int_{0}^{t} \int_{L_{y} L_{z}}^{y} c_{n}^{z}(v) c_{n}(w) \times \\
& \times D_{\Phi V}(x, v, w, T) d w d v d \tau-\frac{x z \pi}{L_{x} L_{y} L_{z}} \sum_{n=1}^{N} n \int_{0}^{t} \int_{L_{x} L_{z}}^{x} \int_{n}^{z}(u) c_{n}(w) D_{\Phi V}(u, v, w, T) d w d u d \tau \times \\
& \times a_{n \Phi V} s_{n}(y) e_{n \Phi V}(t)-\frac{x y \pi}{L_{x} L_{y} L_{z}} \sum_{n=1}^{N} n s_{n}(z) e_{n \Phi V}(t) \int_{0}^{t} \int_{L_{x} L_{y}}^{x} c_{n}(u) c_{n}(v) D_{\Phi V}(u, v, z, T) d v d u d \tau \times \\
& \times a_{n \Phi V}+\frac{x y z}{L_{x} L_{y} L_{z}} \int_{L_{x} L_{y} L_{z}}^{x} \int_{V}^{z} k_{V, V}(u, v, w, T) V^{2}(u, v, w, \tau) d w d v d u+\int_{L_{x} L_{y} L_{z}}^{x} \int_{\Phi V}^{z} f_{\Phi}(u, v, w) d w d v d u \times \\
& \times \frac{x y z}{L_{x} L_{y} L_{z}}-\frac{x y z}{L_{x} L_{y} L_{z}} \int_{L_{x} L_{y} L_{z}}^{x} \int_{V}^{z} k_{V}(u, v, w, T) V(u, v, w, \tau) d w d v d u .
\end{aligned}
$$


We used orthogonality condition of functions of the considered series framework the heterostructure to calculate coefficients $a_{n \Phi \rho}$. The coefficients $a_{n \Phi \rho}$ could be calculated for any quantity of terms $N$. In the common case equations for the required coefficients could be written as

$$
\begin{aligned}
& -\frac{L_{x}^{2} L_{y}^{2} L_{z}^{2}}{\pi^{5}} \sum_{n=1}^{N} \frac{a_{n \Phi I}}{n^{6}} e_{n \Phi I}(t)=-\frac{1}{2 \pi L_{x}} \sum_{n=1}^{N} \int_{0}^{t} \int_{0}^{L_{x}}\left[1-c_{n}(2 x)\right] \int_{0}^{L_{y}}\left\{L_{y}+y s_{n}(2 y)+\frac{L_{y}}{2 \pi n}\left[c_{n}(2 y)-1\right]\right\} \times \\
& \times \frac{a_{n \Phi \Phi}}{n^{2}} \int_{0}^{L_{z}} D_{\Phi I}(x, y, z, T)\left\{z s_{n}(z)+\frac{L_{z}}{2 \pi n}\left[c_{n}(z)-1\right]\right\} d z d y d x e_{n \Phi I}(\tau) d \tau-\frac{1}{2 \pi} \sum_{n=1}^{N} \int_{0}^{t} \int_{0}^{L_{x}}\left\{x s_{n}(2 x)+\right. \\
& \left.+L_{x}+\frac{L_{x}}{2 \pi n}\left[c_{n}(2 x)-1\right]\right\} \int_{0}^{L_{y}}\left[1-c_{n}(2 y)\right] \int_{0}^{L_{z}} D_{\Phi I}(x, y, z, T)\left\{z s_{n}(z)+\frac{L_{z}}{2 \pi n}\left[c_{n}(z)-1\right]\right\} d z d y d x \times \\
& \times a_{n \Phi I} \frac{e_{n \Phi I}(\tau)}{n^{2} L_{y}} d \tau-\frac{1}{2 \pi L_{x}} \sum_{n=1}^{N} \frac{a_{n \Phi I}}{n^{2}} \int_{0}^{t} \int_{0}^{L_{x}}\left\{x s_{n}(x)+\frac{L_{x}}{2 \pi n}\left[c_{n}(x)-1\right]\right\} \int_{0}^{L_{y}}\left\{y s_{n}(2 y)+\frac{L_{y}}{2 \pi n}\left[c_{n}(2 y)-1\right]+\right. \\
& +L_{y} \int_{0}^{L_{z}}\left[1-c_{n}(2 y)\right] D_{\Phi I}(x, y, z, T) d z d y d x e_{n \Phi I}(\tau) d \tau+\frac{1}{\pi^{3}} \sum_{n=1}^{N} \frac{a_{n \Phi}}{n^{3}} \int_{0}^{t} e_{n \Phi I}(\tau) \int_{0}^{L_{x}}\left\{\frac{L_{x}}{2 \pi n}\left[c_{n}(x)-1\right]+\right. \\
& \left.+x s_{n}(x)\right\} \int_{0}^{L_{y}}\left\{y s_{n}(y)+\frac{L_{y}}{2 \pi n}\left[c_{n}(y)-1\right]\right\} \int_{0}^{L_{z}} I^{2}(x, y, z, t) k_{I, I}(x, y, z, T)\left\{\frac{L_{z}}{2 \pi n}\left[c_{n}(z)-1\right]+\right. \\
& \left.+z s_{n}(z)\right\} d z d y d x-\frac{1}{\pi^{3}} \sum_{n=1}^{N} \frac{a_{n \Phi I}}{n^{3}} \int_{0}^{t} e_{n \Phi I}(\tau) \int_{0}^{L_{x}}\left\{x s_{n}(x)+\frac{L_{x}}{2 \pi n}\left[c_{n}(x)-1\right]\right\} \int_{0}^{L_{y}}\left\{\frac{L_{y}}{2 \pi n}\left[c_{n}(y)-1\right]+\right. \\
& \left.+y s_{n}(y)\right\} \int_{0}^{L_{z}}\left\{z s_{n}(z)+\frac{L_{z}}{2 \pi n}\left[c_{n}(z)-1\right]\right\} k_{I}(x, y, z, T) I(x, y, z, t) d z d y d x+\frac{1}{\pi^{3}} \sum_{n=1}^{N} \frac{a_{n \Phi I}}{n^{3}} \times \\
& \times \int_{0}^{t} e_{n \Phi I}(\tau) \int_{0}^{L_{x}}\left\{x s_{n}(x)+\frac{L_{x}}{2 \pi n}\left[c_{n}(x)-1\right]\right\} \int_{0}^{L_{y}}\left\{y s_{n}(y)+\frac{L_{y}}{2 \pi n}\left[c_{n}(y)-1\right]\right\} \int_{0}^{L_{z}}\left\{\frac{L_{z}}{2 \pi n}\left[c_{n}(z)-1\right]+\right. \\
& \left.+z s_{n}(z)\right\} f_{\Phi I}(x, y, z) d z d y d x \\
& -\frac{L_{x}^{2} L_{y}^{2} L_{z}^{2}}{\pi^{5}} \sum_{n=1}^{N} \frac{a_{n \Phi V}}{n^{6}} e_{n \Phi V}(t)=-\frac{1}{2 \pi L_{x}} \sum_{n=1}^{N} \int_{0}^{t} \int_{0}^{L_{x}}\left[1-c_{n}(2 x)\right] \int_{0}^{L_{y}}\left\{L_{y}+y s_{n}(2 y)+\frac{L_{y}}{2 \pi n}\left[c_{n}(2 y)-1\right]\right\} \times \\
& \times \frac{a_{n \Phi V}}{n^{2}} \int_{0}^{L_{z}} D_{\Phi V}(x, y, z, T)\left\{z s_{n}(z)+\frac{L_{z}}{2 \pi n}\left[c_{n}(z)-1\right]\right\} d z d y d x e_{n \Phi V}(\tau) d \tau-\frac{1}{2 \pi} \sum_{n=1}^{N} \int_{0}^{t} \int_{0}^{L_{x}}\left\{x s_{n}(2 x)+\right. \\
& +L_{x}+\frac{L_{x}}{2 \pi n}\left[c_{n}(2 x)-1\right] \int_{0}^{L_{y}}\left[1-c_{n}(2 y)\right] \int_{0}^{L_{z}} D_{\Phi V}(x, y, z, T)\left\{z s_{n}(z)+\frac{L_{z}}{2 \pi n}\left[c_{n}(z)-1\right]\right\} d z d y d x \times \\
& \times a_{n \Phi V} \frac{e_{n \Phi V}(\tau)}{n^{2} L_{y}} d \tau-\frac{1}{2 \pi L_{x}} \sum_{n=1}^{N} \frac{a_{n \Phi V}}{n^{2}} \int_{0}^{t} \int_{0}^{L_{x}}\left\{x s_{n}(x)+\frac{L_{x}}{2 \pi n}\left[c_{n}(x)-1\right]\right\} \int_{0}^{L_{y}}\left\{y s_{n}(2 y)+\frac{L_{y}}{2 \pi n}\left[c_{n}(2 y)-1\right]+\right. \\
& \left.+L_{y}\right\} \int_{0}^{L_{z}}\left[1-c_{n}(2 y)\right] D_{\Phi V}(x, y, z, T) d z d y d x e_{n \Phi V}(\tau) d \tau+\frac{1}{\pi^{3}} \sum_{n=1}^{N} \frac{a_{n \Phi V}}{n^{3}} \int_{0}^{t} e_{n \Phi V}(\tau) \int_{0}^{L_{x}}\left\{\frac{L_{x}}{2 \pi n}\left[c_{n}(x)-1\right]+\right. \\
& \left.+x s_{n}(x)\right\} \int_{0}^{L_{y}}\left\{y s_{n}(y)+\frac{L_{y}}{2 \pi n}\left[c_{n}(y)-1\right]\right\} \int_{0}^{L_{z}} V^{2}(x, y, z, t) k_{V, V}(x, y, z, T)\left\{\frac{L_{z}}{2 \pi n}\left[c_{n}(z)-1\right]+\right.
\end{aligned}
$$




$$
\begin{gathered}
\left.+z s_{n}(z)\right\} d z d y d x-\frac{1}{\pi^{3}} \sum_{n=1}^{N} \frac{a_{n \Phi}}{n^{3}} \int_{0}^{t} e_{n \Phi V}(\tau) \int_{0}^{L_{x}}\left\{x s_{n}(x)+\frac{L_{x}}{2 \pi n}\left[c_{n}(x)-1\right]\right\} \int_{0}^{L_{y}}\left\{\frac{L_{y}}{2 \pi n}\left[c_{n}(y)-1\right]+\right. \\
\left.+y s_{n}(y)\right\} \int_{0}^{L_{z}}\left\{z s_{n}(z)+\frac{L_{z}}{2 \pi n}\left[c_{n}(z)-1\right]\right\} k_{V}(x, y, z, T) V(x, y, z, t) d z d y d x+\frac{1}{\pi^{3}} \sum_{n=1}^{N} \frac{a_{n \Phi V}}{n^{3}} \times \\
\times \int_{0}^{t} e_{n \Phi V}(\tau) \int_{0}^{L_{x}}\left\{x s_{n}(x)+\frac{L_{x}}{2 \pi n}\left[c_{n}(x)-1\right]\right\} \int_{0}^{L_{y}}\left\{y s_{n}(y)+\frac{L_{y}}{2 \pi n}\left[c_{n}(y)-1\right]\right\} \int_{0}^{L_{z}}\left\{\frac{L_{z}}{2 \pi n}\left[c_{n}(z)-1\right]+\right. \\
\left.+z s_{n}(z)\right\} f_{\Phi V}(x, y, z) d z d y d x .
\end{gathered}
$$

\section{DISCUSSION}

We analyzed variation of concentration of dopant in space and time with account variation of concentration of radiation defects in space and time for two cases: (i) implantation of ions of dopant at room temperature; (ii) implantation of ions of dopant at high temperature of considered heterostructure. Fig. 2 shows distributions of concentration of dopant for both considered cases (curves 1 and 2, respectively). The figure shows, that implantation of ions of dopant at high temperature of heterostructure leads to spreading of distributions of concentration of the dopant in comparison with implantation of ions of dopant at room temperature Reason of this situation is increasing of dopant diffusion coefficient with increasing of temperature of technological process.. At the same time one can find acceleration of diffusion of radiation defects from damaged area and acceleration of interaction between these defects (recombination of point defects and generation of their complexes). In this situation annealing time of radiation defects decreases in comparison with the same time after implantation of ions of dopant at room temperature.

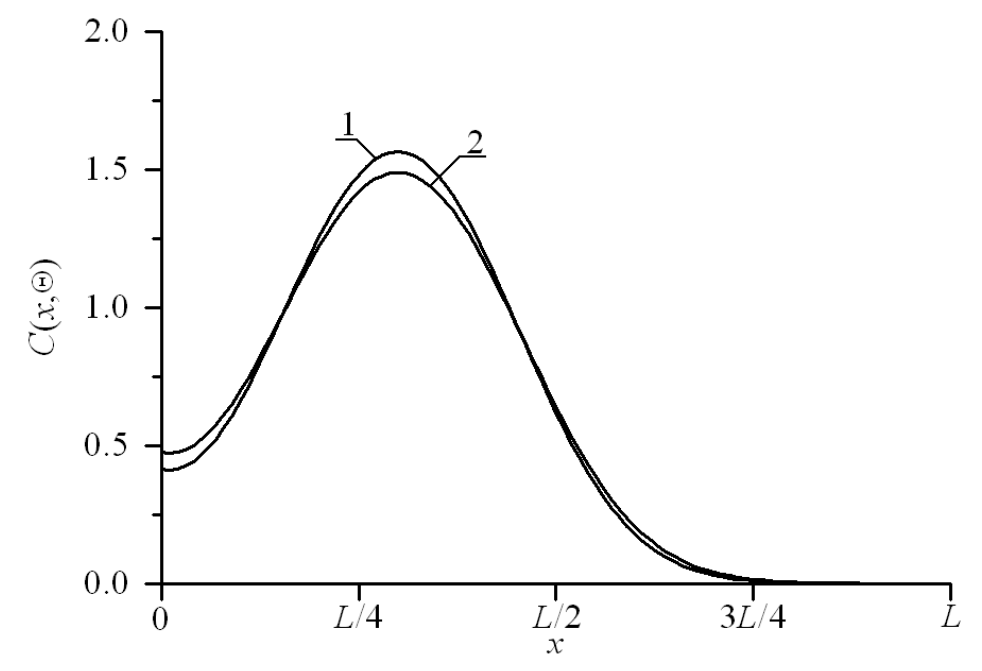

Fig.2. Distributions of concentration of implanted dopant at room temperature (curve 1) and at higher temperature (curve 2)

It should be noted, that using inhomogeneity of heterostructure gives a possibility to increase sharpness of $p-n$-junctions, manufactured near interface of the heterostructure. The sharpness of $p$ - $n$-junctions could be obtained when dopant diffusion coefficient in the epitaxial layer is larger, than in the substrate. In this situation one can find acceleration of dopant diffusion in doped area during annealing of radiation defects and deceleration of dopant diffusion in nearest area during the annealing. The relation between values of dopant diffusion coefficient gives a possibility to use semiinsulating property of interface between layers of heterostructure. At the same time ho- 
mogeneity of distribution of implanted dopant increases (see Fig. 3). However in the case optimization of annealing attracted an interest [16-20]. The optimization has been done framework recently introduced criterion [16-20].

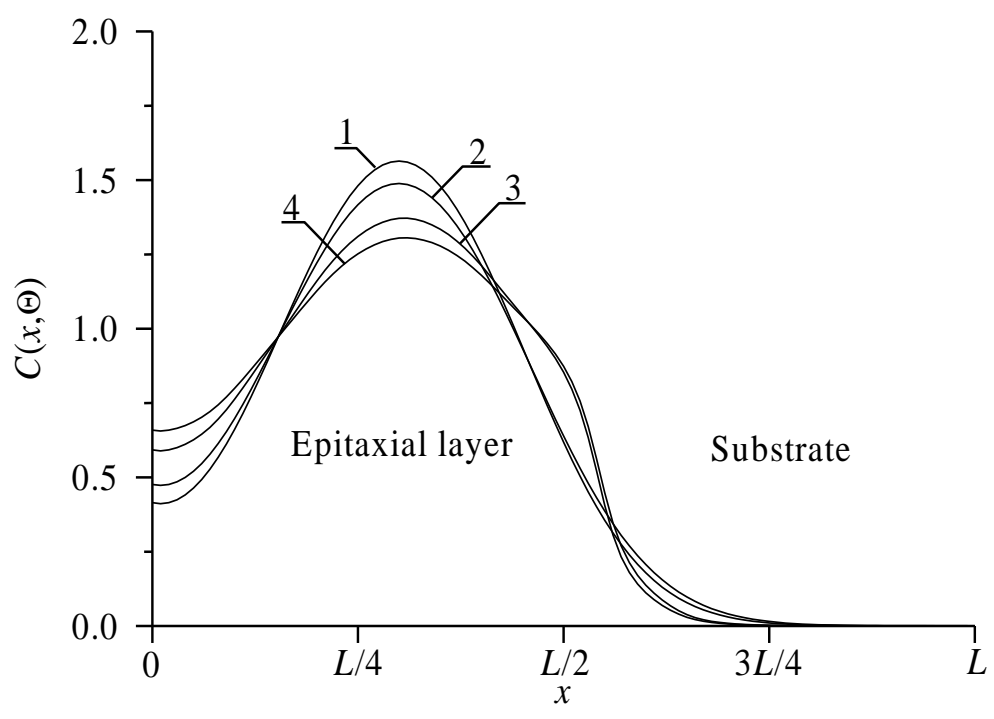

Fig.3. Dependences of concentration of implanted dopant on coordinate in direction, which is perpendicular to interface between epitaxial layer substrate. Curves 1 and 3 corresponds to annealing time $\Theta=0.0048\left(L_{x}{ }^{2}+L_{y}{ }^{2}+L_{z}{ }^{2}\right) / D_{0}$. Curves 2 and 4 corresponds to annealing time $\Theta=$ $0.0057\left(L_{x}{ }^{2}+L_{y}{ }^{2}+L_{z}{ }^{2}\right) / D_{0}$. Curves 1 and 2 are distributions of concentration of dopant in homogenous sample. Curves 3 and 4 are distributions of concentration of dopant in heterostructure

\section{Conclusions}

In this paper we analyzed manufacturing of implanted-junction rectifiers at different temperatures. We obtain, that increasing of temperature of doping leads to increasing of spreading of distribution of concentration of dopant an at the same time to decreasing of quantity of radiation defects. It has been shown, that variation of the temperature of heterostructure during the ion implantation gives a possibility to accelerate the technological process. We introduce an approach to decrease continuance of technological process.

\section{ACKNOWLEDGEMENTS}

This work is supported by the agreement of August 27, 2013 № 02.B.49.21.0003 between The Ministry of education and science of the Russian Federation and Lobachevsky State University of Nizhni Novgorod, educational fellowship for scientific research of Government of Russia, educational fellowship for scientific research of Government of Nizhny Novgorod region of Russia and educational fellowship for scientific research of Nizhny Novgorod State University of Architecture and Civil Engineering.

\section{REFERENCES}

[1] V.I. Lachin, N.S. Savelov. Electronics (Phoenix, Rostov-na-Donu, 2001).

[2] A.G. Alexenko, I.I. Shagurin. Microcircuitry (Radio and communication, Moscow, 1990).

[3] N.A. Avaev, Yu.E. Naumov, V.T. Frolkin. Basis of microelectronics (Radio and communication, Moscow, 1991).

[4] D. Fathi, B. Forouzandeh. Accurate analysis of global interconnects in nano-FPGAs. Nano. Vol. 4 (3). P. 171-176 (2009). 
[5] D. Fathi, B. Forouzandeh, N. Masoumi. New enhanced noise analysis in active mixers in nanoscale technologies. Nano. Vol. 4 (4). P. 233-238 (2009).

[6] S.A. Chachuli, P.N.A. Fasyar, N. Soin, N.M. Karim, N. Yusop. Pareto ANOVA analysis for CMOS $0.18 \mu \mathrm{m}$ two-stage Op-amp. Mat. Sci. Sem. Proc. Vol. 24. P. 9-14 (2014).

[7] A.O. Ageev, A.E. Belyaev, N.S. Boltovets, V.N. Ivanov, R.V. Konakova, Ya.Ya. Kudrik, P.M. Litvin, V.V. Milenin, A.V. Sachenko. Au-TiBx-n-6H-SiC Schottky barrier diodes: the features of current flow in rectifying and nonrectifying contacts Semi-conductors. Vol. 43 (7). P. 897-903 (2009).

[8] Jung-Hui Tsai, Shao-Yen Chiu, Wen-Shiung Lour, Der-Feng Guo. High-performance InGaP/GaAs pnp $\delta$-doped heterojunction bipolar transistor Semiconductors. Vol. 43 (7). P.971-974 (2009).

[9] M.J. Kumar, T.V. Singh. Quantum confinement effects in strained silicon MOSFETs MOSFETs. Int. J. Nanoscience. Vol. 7 (2-3). P. 81-84 (2008).

[10] P. Sinsermsuksakul, K. Hartman, S.B. Kim, J. Heo, L. Sun, H.H. Park, R. Chakraborty, T. Buonassisi, R.G. Gordon. Enhancing the efficiency of $\mathrm{SnS}$ solar cells via band-offset engineering with a zinc oxysulfide buffer layer. Appl. Phys. Lett. Vol. 102 (5). P. $053901-053905$ (2013).

[11] V.V. Kozlivsky. Modification of semiconductors by proton beams (Nauka, Sant-Peterburg, 2003, in Russian).

[12] Z.Yu. Gotra. Technology of microelectronic devices (Radio and communication, Moscow, 1991).

[13] V.L. Vinetskiy, G.A. Kholodar', Radiative physics of semiconductors. ("Naukova Dumka", Kiev, 1979, in Russian).

[14] P.M. Fahey, P.B. Griffin, J.D. Plummer. Point defects and dopant diffusion in silicin. Rev. Mod. Phys. Vol. 61. № 2. P. 289-388 (1989).

[15] M.L. Krasnov, A.I. Kiselev, G.I. Makarenko. Integral equations ("Science", Moscow, 1976).

[16] E.L. Pankratov. Decreasing of depth of implanted-junction rectifier in semiconductor heterostructure by optimized laser annealing. J. Comp. Theor. Nanoscience. Vol.7 (1). P. 289-295 (2010).

[17] E.L. Pankratov, E.A. Bulaeva. Doping of materials during manufacture p-n-junctions and bipolar transistors. Analytical approaches to model technological approaches and ways of optimization of distributions of dopants. Reviews in Theoretical Science. Vol. 1 (1). P. 58-82 (2013).

[18] E.L. Pankratov, E.A. Bulaeva. Doping of materials during manufacture p-n-junctions and bipolar transistors. Analytical approaches to model technological approaches and ways of optimization of distributions of dopants. J. Comp. Theor. Nanoscience. Vol. 12 (6). P. 976-990 (2014).

[19] E.L. Pankratov, E.A. Bulaeva. An approach to manufacture of bipolar transistors in thin film structures. On the me-thod of optimization. Int. J. Micro-Nano Scale Transp. Vol. 4 (1). P. 17-31 (2014).

[20] E.L. Pankratov, E.A. Bulaeva. An approach to decrease dimensions of field-effect transistors without p-n-junctions. Int. J. Mod. Phys. B. Vol. 28 (27). P. 1450190-1-1450190-17 (2014)

\section{AUTHORS}

Pankratov Evgeny Leonidovich was born at 1977. From 1985 to 1995 he was educated in a secondary school in Nizhny Novgorod. From 1995 to 2004 he was educated in Nizhny Novgorod State University: from 1995 to 1999 it was bachelor course in Radiophysics, from 1999 to 2001 it was master course in Radiophysics with specialization in Statistical Radiophysics, from 2001 to 2004 it was PhD course in Radiophysics. From 2004 to 2008 E.L. Pankratov was a leading technologist in Institute for Physics of Microstructures. From 2008 to 2012 E.L. Pankratov was a senior lecture/Associate Professor of Nizhny Novgorod State University of Architecture and Civil Engineering. 2012-2015 Full Doctor course in Radiophysical Department of Nizhny Novgorod State University. Since 2015 E.L. Pankratov is an Associate Professor of Nizhny Novgorod State University. He has 135 published papers in area of his researches.

Bulaeva Elena Alexeevna was born at 1991. From 1997 to 2007 she was educated in secondary school of village Kochunovo of Nizhny Novgorod region. From 2007 to 2009 she was educated in boarding school "Center for gifted children". From 2009 she is a student of Nizhny Novgorod State University of Architecture and Civil Engineering (spatiality "Assessment and management of real estate"). At the same time she is a student of courses "Translator in the field of professional communication" and "Design (interior art)" in the University. Since 2014 E.A. Bulaeva is in a PhD program in Radiophysical Department of Nizhny Novgorod State University. She has 90 published papers in area of her researches. 